\title{
Temperaturas decadais extremas e média em São Bento do Una - Pernambuco,
}

\section{Brasil}

\author{
Extreme and average decade temperatures in São Bento do Una - Pernambuco, Brazil \\ Temperaturas extremas y promedio por décadas en São Bento do Una - Pernambuco, Brasil
}

Recebido: 09/03/2021 | Revisado: 16/03/2021 | Aceito: 05/04/2021 | Publicado:15/04/2021

\author{
Raimundo Mainar de Medeiros \\ ORCID: https://orcid.org/0000-0003-3455-9876 \\ Universidade Federal Rural de Pernambuco, Brasil \\ E-mail: mainarmedeiros@gmail.com \\ Romildo Morant de Holanda \\ ORCID: https://orcid.org/0000-0001-7945-3616 \\ Universidade Federal Rural de Pernambuco, Brasil \\ E-mail: romildomorant@gmail.com \\ Luciano Marcelo Fallé Saboya \\ ORCID: https://orcid.org/0000-0002-7586-6867 \\ Universidade Federal de Campina Grande, Brasil \\ E-mail: 1saboya@hotmail.com \\ Wagner Rodolfo de Araújo \\ ORCID: https://orcid.org/0000-0001-7203-0338 \\ Universidade Estácio de Sá, Brasil \\ E-mail: wagneraraujops@gmail.com \\ Manoel Viera de França \\ ORCID: https://orcid.org/0000-0003-4973-9327 \\ Universidade Federal Rural de Pernambuco, Brasil \\ E-mail: manoelvieira.ufrpe@gmail.com \\ Fernando Cartaxo Rolim Neto \\ ORCID: https://orcid.org/0000-0002-6411-2058 \\ Universidade Federal Rural de Pernambuco, Brasil \\ E-mail: fernandocartaxo@yahoo.com.br
}

\begin{abstract}
Resumo
As temperaturas extremas do ar são mutáveis ao percurso do tempo e regiões e possui variação anual quanto às suas características, a análise de períodos curtos, desde que realizados para anos sucessivos de no mínimo dez anos, proporcionar avaliar o comportamento do clima da área. Tem-se como objetivo avaliar, temporal e espacialmente, as tendências climáticas, anuais e estacionais, da temperatura extremam e média para São Bento do Una - Pernambuco entre 1950-2020, bem como discutir possíveis causas. Espera-se que essas informações possam subsidiar pesquisadores e as autoridades governamentais em diversas áreas de atividade, especialmente na agricultura e avicultura. Os dados térmicos extremos e média foram gerados pelo software Estima_T (1950-2020) e dividiu-se os referidos dados a cada 10 anos. Utilizou-se da estatística básica para calcular as médias e outros parâmetros relevantes ao desenvolvimento do estudo. Os dados das flutuabilidades térmicas do ar auxiliam as tomadas de decisões do meio agropecuário, agronegócio urbano e rural em múltiplas áreas do conhecimento. A temperatura média é a representação da variabilidade e suas oscilações das temperaturas extremas, qualquer variabilidade nestes elementos as temperaturas médias se adequam as suas oscilações dos sistemas sinóticos atuantes na quadra chuvosa e seco tal como dos impactos no meio ambiente e a sua elevação em relação ao nível do mar. Variabilidades climáticas registradas em escala global, regional e local foram constatadas na área estudada, para sessenta e sete anos de dados.
\end{abstract}

Palavras-chave: Variáveis atmosféricas; Variabilidade climática; Flutuações térmicas.

\section{Abstract}

Extreme air temperatures are changeable over time and regions and have an annual variation in their characteristics, the analysis of short periods, provided that they are carried out for successive years of at least ten years, provide an assessment of the area's climate behavior. The objective is to evaluate, temporally and spatially, the climatic trends, annual and seasonal, of extreme and average temperature for São Bento do Una - Pernambuco between 1950-2020, as well as to discuss possible causes. It is hoped that this information will be able to subsidize researchers and government authorities in several areas of activity, especially in agriculture and poultry. The extreme and average thermal data were generated by the Estima_T software (1950-2020) and the data was divided every 10 years. Basic statistics were used to calculate the means and other parameters relevant to the development of the study. The data on 
Research, Society and Development, v. 10, n. 4, e40110413726, 2021

(CC BY 4.0) | ISSN 2525-3409 | DOI: http://dx.doi.org/10.33448/rsd-v10i4.13726

thermal air buoyancy aids decision-making in the agricultural, urban and rural agribusiness in multiple areas of knowledge. The average temperature is the representation of the variability and its oscillations of the extreme temperatures, any variability in these elements the average temperatures adapt to their oscillations of the synoptic systems acting in the rainy and dry season as well as the impacts on the environment and its elevation in relation sea level. Climatic variability recorded on a global, regional and local scale was found in the studied area, for sixty-seven years of data.

Keywords: Atmospheric variables; Climatic variability; Thermal fluctuations.

\section{Resumen}

Las temperaturas extremas del aire son cambiantes en el tiempo y las regiones y tienen una variación anual en sus características, el análisis de períodos cortos, siempre que se realicen durante años sucesivos de al menos diez años, proporcionan una evaluación del comportamiento climático del área. El objetivo es evaluar, temporal y espacialmente, las tendencias climáticas, anuales y estacionales, de la temperatura media y extrema para São Bento do Una Pernambuco entre 1950-2020, así como discutir las posibles causas. Se espera que esta información pueda subsidiar a investigadores y autoridades gubernamentales en varias áreas de actividad, especialmente en la agricultura y la avicultura. Los datos térmicos extremos y promedio fueron generados por el software Estima_T (1950-2020) y los datos se dividieron cada 10 años. Se utilizaron estadísticas básicas para calcular las medias y otros parámetros relevantes para el desarrollo del estudio. Los datos sobre flotabilidad del aire térmico ayudan a la toma de decisiones en la agroindustria agrícola, urbana y rural en múltiples áreas del conocimiento. La temperatura media es la representación de la variabilidad y sus oscilaciones de las temperaturas extremas, cualquier variabilidad en estos elementos las temperaturas medias son adecuadas a sus oscilaciones de los sistemas sinópticos que actúan en la época lluviosa y seca así como a los impactos sobre el medio ambiente y su elevación en relación con el nivel del mar. Se encontró variabilidad climática registrada a escala global, regional y local en el área estudiada, durante sesenta y siete años de datos.

Palabras clave: Variables atmosféricas; Variabilidad climática; Fluctuaciones térmicas.

\section{Introdução}

A temperatura do ar, especificamente aquela medida à superfície, em valores máximos, médios e mínimos, é parâmetro importante para diversas áreas geográficas. Sua dinâmica é influenciada por variações espaciais e temporais, que determinam a formação de ambientes termicamente homogêneos ou contrastantes.

Temperaturas extremas (máxima - mínima) desde 1950 vêm sendo acrescentada com frequência e intensidade em diversas áreas do globo. As tendências positivas de acréscimo cobrem áreas de maiores tendências negativas (Collins, Chaves \& Marques, 2009; Reguero, Losada \& Mendez 2019). Estudos estão sendo desenvolvidos visando compreender as oscilações climáticas que ocorrem nas regiões: Amazônica (Lopart et al., 2018), Bacia hidrográfica do rio Prata (Bertoli, 2018; Montroull, Saurral \& Camilloni, 2018), Sul do continente Americano (Cordeiro, Berlato, Foutana \& Alves, 2016; Kayano, Andreoli, Souza \& Garcia 2017; Detzer et al., 2019), Sudeste e Nordeste do Brasil. (Coelho et al., 2016; Oliveira, Santos \& Lima 2017). Assim como, estudos nos estados do Espirito Santo (Feitoza, Seardua, Sediyama \& Vall, 1980), Região Nordeste do Brasil (Cavalcanti, Silva \& Souza 2006; Cavalcanti \& Silva 1994) e sobre os modelos de regressão múltipla abrangendo, sobretudo, estados e regiões (Oliveira Neto, Santos \& Lima 2000), para fins de zoneamento agroclimatológicos (Barbarisi, et al., 2006; Bardin, Junior \& Morais, 2010) onde se utilizam da estimativa da temperatura.

Medeiros \& Cavalcanti (2020) estudando as tendências térmicas máximas e mínimas anuais climáticas para Bom Jesus Piauí, (1960-2018) onde discutiram sua provável origem. Os autores realizaram uma delimitação do comportamento climático, fornecendo limitações de fases quente e/ou frio, a qual servira de alerta as autoridades e aos tomadores de decisões, em realizarem melhores planejamentos agropecuários e dos agronegócios. Concluíram que a caracterização temporal, em escala mensal e anual, gerou estimações simuladas próximas aos valores observados, fato comprovado pelos índices estatísticos de atuação satisfatórios.

Medeiros (2018) revelou que as oscilações nas amplitudes térmicas para a grande metrópole Recife esta materializada em ganhos na agropecuária, agronegócio, saúde, educação, moradia e na qualidade de vida que faz referência à satisfação de 
Research, Society and Development, v. 10, n. 4, e40110413726, 2021

(CC BY 4.0) | ISSN 2525-3409 | DOI: http://dx.doi.org/10.33448/rsd-v10i4.13726

necessidades, tanto básicas quanto não básicas, da população. As flutuabilidades crescentes na amplitude térmica vêm ocorrendo pela falta de planejamento das cidades, de arborização e das altas incidências de queimadas e desmatamento.

Segundo as estimativas do Centro de Monitoramento de Deslocamento Interno (IDMC, 2017), até 2050 o número de refugiados por desastres climáticos chegará a um bilhão de pessoas. Recentemente, registraram-se deslocamentos por desastres climáticos - e eles não são poucos: algo em torno de 25 milhões de pessoas já deixou seus países devido a problemas ambientais como secas, inundações, tempestades e incêndios florestais.

Medeiros, Kozmhinsy, Holanda \& Silva (2018) mostraram que os resultados das oscilações térmicas estudadas estão relacionados a elevação e a latitude, sendo uma das variáveis fisiográficas que melhor explicam as flutuações térmicas mensal e anual em estudo. As oscilações térmicas médias foram decorrentes dos sistemas sinóticos predominantes dos períodos chuvosos e secos, assim como dos impactos ao meio ambiente. Registraram-se reduções térmicas em conformidade com o deslocamento da quadra chuvosa e das atuações dos efeitos regionais e locais.

É consenso, entre os cientistas da área de meteorologia e afins, que as tendências climáticas observadas, especialmente a partir da segunda metade do século XX, aumentaram significativamente. Segundo o Painel Intergovernamental de Mudanças Climáticas (IPCC), essas tendências observadas no passado recente apresentam alta probabilidade de continuarem no mesmo sentido no século XXI (IPCC, 2007; IPCC, 2014).

Medeiros et al. (2012) efetuaram o cálculo para a temperatura média diária empregando diferentes metodologias para os municípios de Parnaíba, Picos e Gilbués localizados no Estado do Piauí Utilizaram cinco métodos para o cálculo da temperatura média diária do ar, adotando como padrão o recomendado pelo Instituto Nacional de Meteorologia (INMET). Os quatro métodos avaliados em relação ao padrão possuíram desempenho classificado como "Muito bom e Ótimo", com índice de confiança variando entre 0,83 a 0,98 . Os resultados indicam que na região estudada os quatro métodos avaliados podem ser utilizados nas estimativas das temperaturas médias diárias do ar.

Matos, Medeiros, Silva \& Saboya (2015) usaram dados térmicos mensais para o município de Barbalha (CE) e comprovaram que a elevação e a latitude são as variáveis fisiográficas que explicam com maior perfeição a variação da temperatura do ar anual.

Medeiros, Borges \& Gomes Filho (2014) estudando as oscilações da temperatura máxima, umidade relativa e precipitação para Brasília, notaram variabilidades nestes elementos climáticos com tendências a mudanças e afirmam que a redução nos índices de umidade e as taxas de aumentos na temperatura e precipitações são decorrentes dos efeitos locais os quais pode estar relacionado com o aumento da temperatura, que faz com que se tenha uma maior evaporação e consequentemente maior incidência pluvial.

Tem-se como objetivo analisar, temporal e espacialmente, as tendências climáticas, anuais e estacionais, da temperatura máxima, média e mínima, do ar por décadas e seu comparativo com a média climática para São Bento do Una PE, no período de sessenta e sete anos (1950-2020), bem como discutir possíveis causas. Espera-se que essas informações possam subsidiar pesquisadores e tomadores de decisão em diversas áreas de atividade, especialmente na agropecuária e avicultura.

\section{Metodologia}

São Bento do Una posiciona-se na mesorregião Agreste e na Microrregião do Vale do Ipojuca Pernambucano, o município tem Belo Jardim ao norte, sul Jucati, Jupi e Lajedo, leste Cachoeirinha, e a oeste Capoeiras, Sanharó e Pesqueira. A 
sede do município tem altitude de 614 metros e coordenadas geográficas de $08^{\circ} 31^{\prime} 22^{\prime \prime}$ de latitude sul e $36^{\circ} 06^{\prime} 40^{\prime \prime}$ de longitude oeste.

Figura 1 - Posicionamento de São Bento do Una dentro do estado Pernambucano.

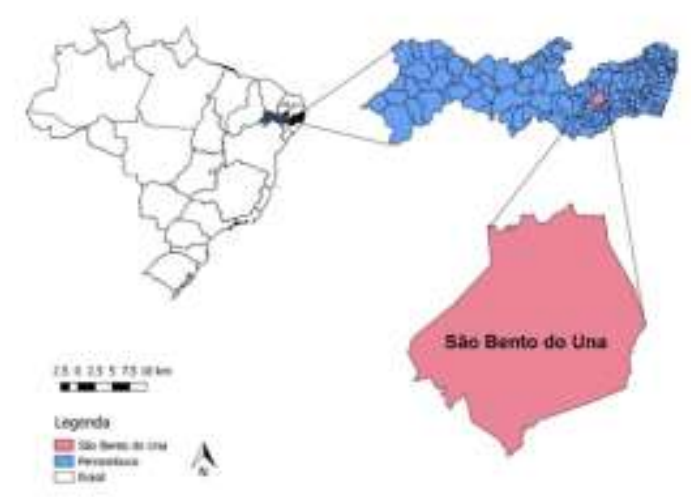

Fonte: Medeiros, (2021).

Seu clima é do tipo "As" (Tropical Chuvoso, com verão seco), (Medeiros, Holanda \& Silva 2018; Alvares et al. 2014). A quadra chuvosa se inicia em fevereiro com chuvas de pré-estação (chuvas que ocorrem antes da quadra chuvosa) com seu término ocorrendo no mês de agosto e podendo se prolongar até setembro. O trimestre chuvoso centra-se de maio, junho e julho e os meses seco ocorrem entre outubro, novembro e dezembro. Os fatores causadores de chuvas no município são a contribuição da Zona de Convergência Intertropical (ZCIT), formação dos vórtices ciclônicos de altos níveis (VCAS), contribuição dos ventos alísios de nordeste no transporte de vapor e umidade a quais condensam e forma nuvens provocando chuvas de moderadas a fortes intensidades, linhas de instabilidades, orografia e suas contribuições local e regional em conformidade com Medeiros (2016).

Os dados de temperaturas extremas e média foram estimados pelo software Estima_T (Cavalcanti, Silva \& Souza, 1994; Cavalcanti \& Silva, 2006) dada por:

$$
\mathrm{T}=\mathrm{C}_{0}+\mathrm{C}_{1} \lambda+\mathrm{C}_{2} \varnothing+\mathrm{C}_{3} \mathrm{~h}+\mathrm{C}_{4} \lambda^{2}+\mathrm{C}_{5} \varnothing^{2}+\mathrm{C}_{6} \mathrm{~h}^{2}+\mathrm{C}_{7} \lambda \varnothing+\mathrm{C}_{8} \lambda \mathrm{h}+\mathrm{C}_{9} \varnothing \mathrm{h}
$$

Em que:

$\mathrm{C}_{0}, \mathrm{C}_{1}, \ldots . ., \mathrm{C}_{9}$ são constantes;

$\lambda, \lambda^{2}, \lambda \varnothing, \lambda \mathrm{h}$ longitude;

$\varnothing, \varnothing^{2}, \lambda \varnothing$ latitude;

$\mathrm{h}, \mathrm{h}^{2}, \lambda \mathrm{h}, \varnothing \mathrm{h}$ altura.

Os dados obtidos pelo software correspondem ao período de 1950-2020, foram geradas por planilhas eletrônicas e realizam-se os cálculos básicos estatísticos para o desenvolvimento deste trabalho. Os dados térmicos máximo, médio e mínimo foram estimados para o período 1950-2020 e dividiram-se os referidos dados a cada 10 anos. Utilizou-se da estatística básica para calcular as médias e outros parâmetros relevantes ao desenvolvimento do estudo. 
Research, Society and Development, v. 10, n. 4, e40110413726, 2021

(CC BY 4.0) | ISSN 2525-3409 | DOI: http://dx.doi.org/10.33448/rsd-v10i4.13726

\section{Resultados e Discussão}

Neste estudo chamamos a atenção dos leitores para a metodologia de analise. Trabalhou-se com as médias decadais de (1950-1959; 1960-1969; 1970-1979; 1980-1989; 1990-1999; 2000-2009 e 2010-2020), para as temperaturas máximas, médias e mínimas das referidas décadas e seu comparativo utilizou-se das medias das três temperaturas referentes ao período de 19502020 .

O meio ambiente é constituído por um conjunto natural de componentes (a)bióticos em constantes e complexas interações. Nessas relações mútuas, o clima atua como resultante dessas influências recíprocas. Nos dias atuais presenciamos a ocorrência de eventos atmosféricos extremos. As informações das variáveis meteorológicas são de fundamental importância para a elaboração de planejamento e de combate as graves consequências associadas a esses elementos.

As temperaturas climáticas superaram as temperaturas decadal (1950-1959), nas oscilações das temperaturas máximas, médias e mínimas com aumento de $0,2^{\circ} \mathrm{C}$ em todos os meses e nos valores anuais (Figura 2). Estas variabilidades foram decorrentes dos sistemas meteorológicos de meso e micro escala regional e local e da cobertura do solo visto que nesta década ainda não se tinha agricultura em expansão e a avicultura.

Figura 2 - Temperatura decadal (1950-1959) e seu comparativo com a da série 1950-2019 em São Bento do Una - PE.

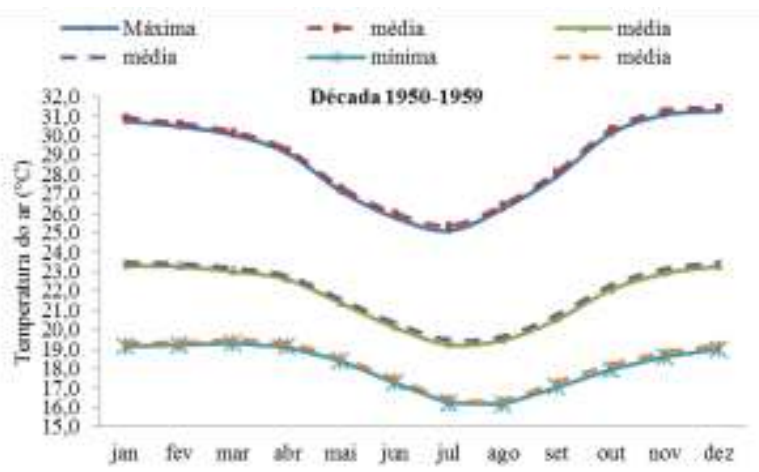

Fonte: Medeiros, (2021).

Com a expansão da agricultura e o inicio da formação da avicultura, registrou-se o desmatamento em larga escala. Na Figura 3 registram-se oscilações térmicas decadal (1960-1969) e seu comparativo com a média climática (1950-2020) para São Bento do Una - PE. As temperaturas médias da máxima, média e mínima superaram as flutuações térmicas decendiais (19601969). As variabilidades das temperaturas mínimas foram as que mais sofreram oscilações tais variabilidades (Rossato, 2011). 
Research, Society and Development, v. 10, n. 4, e40110413726, 2021

(CC BY 4.0) | ISSN 2525-3409 | DOI: http://dx.doi.org/10.33448/rsd-v10i4.13726

Figura 3 - Temperatura decadal (1960-1969) e seu comparativo com a da série 1950-2020 em São Bento do Una - PE.

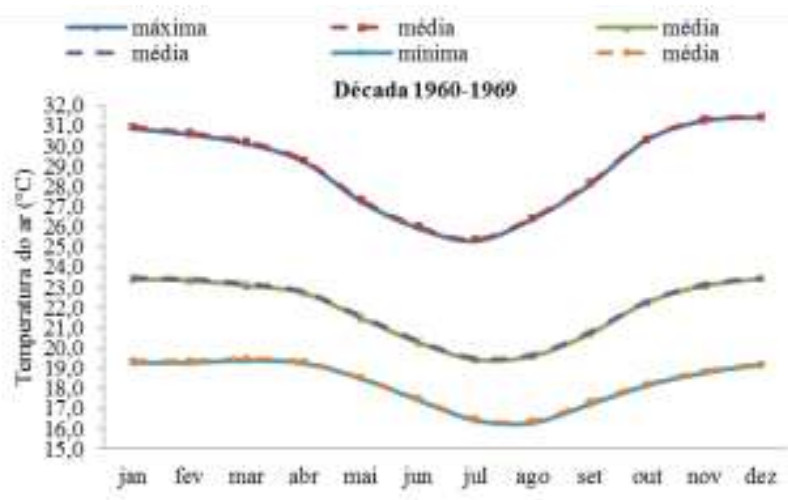

Fonte: Medeiros, (2021).

Araújo \& Gonçalves, (1998) observaram que em média as temperaturas mínimas vêm elevando-se, o que indica uma redução no grau de rigorosidade de inverno. O autor ainda comenta que em média os invernos vêm registrando aquecimento, entretanto os eventos extremos da temperatura mínima têm ocorrido com menor frequência. Estes pesquisadores observaram que a amplitude dos invernos tem diminuído, pois existe um evidente aumento nas temperaturas mínimas em contraposição a um comportamento estacionário das temperaturas máximas. Os autores analisaram o comportamento da temperatura mínima e observaram tendência de aumento.

Junho e julho registraram os menores valores da temperatura máxima, média e mínima para a década de 1970-1979 com redução de $0,2^{\circ} \mathrm{C}$. Outubro, novembro, dezembro e janeiro registraram-se os valores mais altos de temperatura. Destaca agosto com oscilações de temperatura mínima que registrar o menor valor da serie em estudo. Estas reduções em agosto foram decorrentes das atividades dos sistemas locais seguidos de chuvas isoladas pela madrugada. (Figura 4).

Figura 4 - Temperatura decadal (1970-1979) e seu comparativo com a da série 1950-2020 em São Bento do Una - PE.

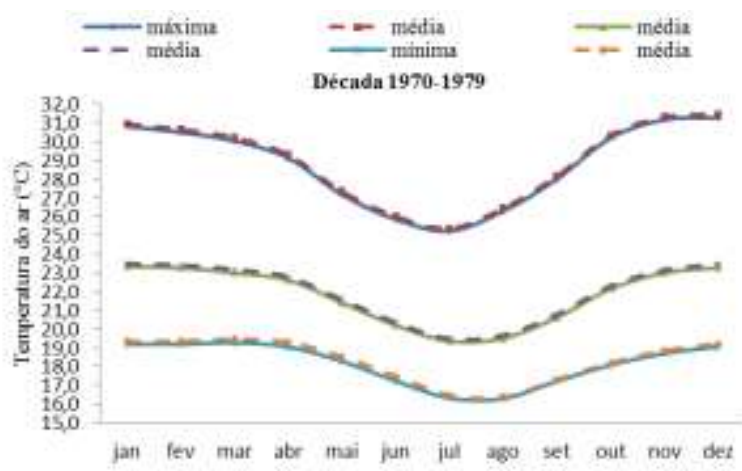

Fonte: Medeiros, (2021).

Campos (2010) afirmaram que a variabilidade da temperatura do ar nas duas últimas décadas tem se destacado pelas elevadas anomalias de temperatura média global do ar (Climate Research Unit, 2009). Entre o período de1961 a 1990 , o ano 1998 foi o mais quente com $0,55^{\circ} \mathrm{C}$ acima da temperatura média anual. $\mathrm{O}$ ano de 2009 foi o sexto ano mais quente, com exceção dos anos de 1998, 2005, 2003, 2002, 2004. 
Vincent et al. (2005) mostraram que existe a tendência expressiva de aumento da temperatura mínima, aumento de noites quentes e redução da amplitude diária em toda a América do Sul, especialmente no Sudeste da América do Sul, estes resultados vêm a corroborar com o presente estudo.

As variabilidades das temperaturas máximas, médias e mínimas e seus comparativos com as médias climáticas para a década de 1980-1989 podem ser visualizados na Figura 5.

A temperatura máxima decadal oscilou entre $25,5^{\circ} \mathrm{C}$ em julho a $31,6^{\circ} \mathrm{C}$ em dezembro, a temperatura média anual da década foi de $29,1^{\circ} \mathrm{C}$. A temperatura climatológica da série $1950-2020$ foi reduzida de $0,1^{\circ} \mathrm{C}$. A mesma redução foi registrada para as temperaturas médias e temperaturas mínimas.

Figura 5 - Temperatura decadal (1980-1989) e seu comparativo com a da série 1950-20120 em São Bento do Una - PE.

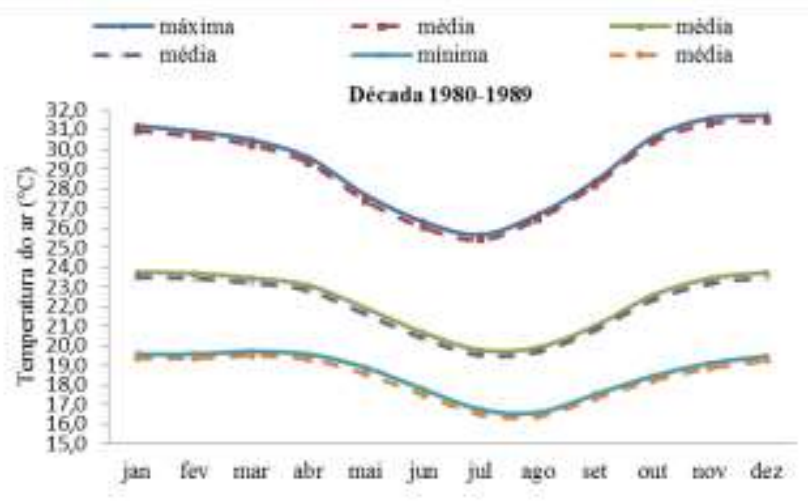

Fonte: Medeiros, (2021).

Melo, Medeiros \& Souza (2015) analisaram a flutuação climática da temperatura máxima, média e mínima do estado de Pernambuco, enfocando tais variações como um meio para compreender futuras mudanças. Usaram dados térmicos anuais no período de 1960 a 1990. Afirmaram que o conhecimento do comportamento climático de uma região sendo de fundamental importância para estudos de previsão do tempo e principalmente para o planejamento agropecuário, contribuindo com informações ao homem do campo para não realizar queimadas na hora do preparo das terras para o plantio, evitando desta forma que aumento do referido parâmetro ocorra. A delimitação do período mais quente ou mais frio serve de alerta às autoridades federais, estaduais e municipais e tomadores de decisões, com objetivo de melhor planejamento. Este estudo corrobora com resultados encontrados no presente estudo.

Figura 6 observa-se as flutuações das temperaturas decadais (1990-1999) máximas, média e mínima e seu comparativo com a temperatura climatológica (1950-2020) para São Bento do Una - PE. As flutuações das temperaturas máximas, médias e mínimas das décadas fluíram acima das suas médias. As temperaturas máximas anuais dos três elementos analisados foram de: $29,2^{\circ} \mathrm{C}$ decadal e sua climatológica com $29^{\circ} \mathrm{C}$, os flutuações das temperaturas médias foram de $22,2^{\circ} \mathrm{C}$ e $22^{\circ} \mathrm{C}$ respectivamente as temperaturas mínimas e suas climatológicas foram de $18,6^{\circ} \mathrm{C}$ e $18,3^{\circ} \mathrm{C}$.

O IPCC (2007) afirma que onze dos doze anos anteriores a 2006 estão entre os anos mais quentes desde o período de 1850 , sendo que a temperatura média global aumentou linearmente aproximadamente $+0,1^{\circ} \mathrm{C}$ por década nos últimos 50 anos (1957 a 2006). 
Research, Society and Development, v. 10, n. 4, e40110413726, 2021

(CC BY 4.0) | ISSN 2525-3409 | DOI: http://dx.doi.org/10.33448/rsd-v10i4.13726

Figura 6 - Temperatura decadal (1990-1999) e seu comparativo com a da série 1950-2020 em São Bento do Una - PE.

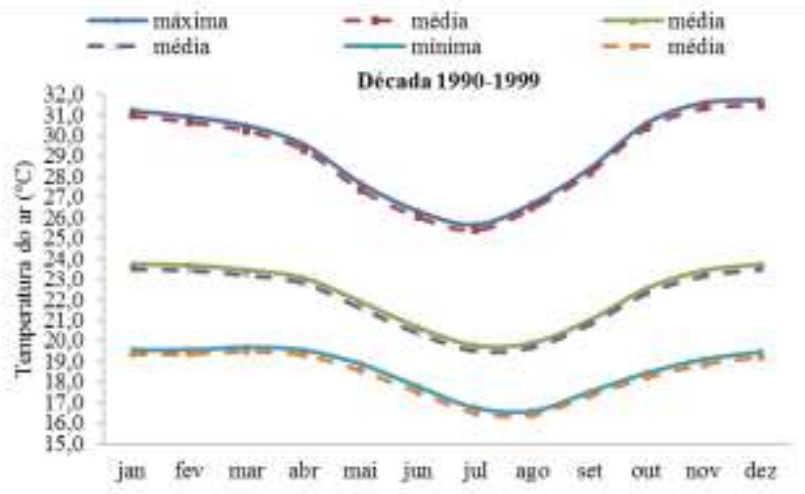

Fonte: Medeiros, (2021).

Rossato (2011) localizou tendência de aumento linear das temperaturas mínimas médias $\left(0,05\right.$ a $0,14^{\circ} \mathrm{C}$ no período de 1970 a 2007) e nas análises polinomiais das estações do Rio Grande do Sul que contribuem para a tendência positiva da temperatura mínima do ar.

As oscilações das temperaturas máximas, médias e mínimas para a década de 2000-2009 e seus respectivos comparativos podem ser observadas na Figura 7. Nas temperaturas máximas e médias decadais superaram as médias climatológicas. A temperatura mínima apresentou oscilações de igualdade (junho a março) e aumento entre os meses de abril e maio na sua climatologia, estas flutuações estão em concordância com o IPCC (2007) e Marengo \& Silva (2006) e vem a corroborar com os resultados desta pesquisa.

Figura 7 - Temperatura decadal (2000-2009) e seu comparativo com a da série 1950-2020 em São Bento do Una - PE.

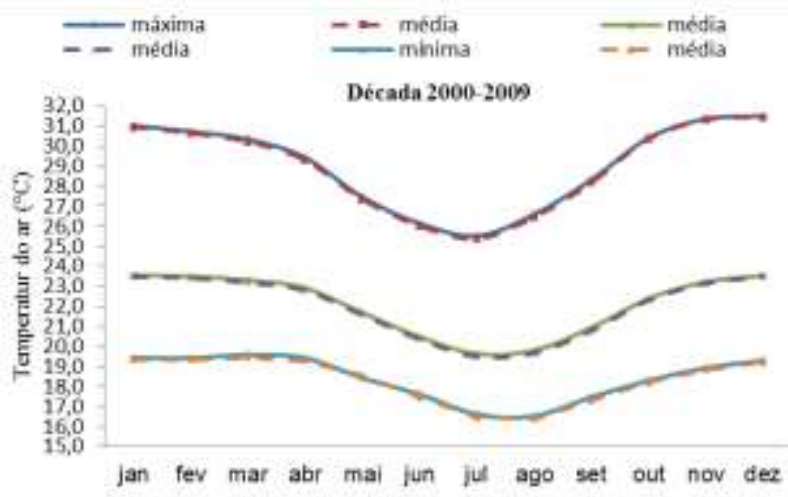

Fonte: Medeiros, (2021).

Na Figura 8 têm-se as flutuações da temperatura do período de 2010-2020 em São Bento do Una - PE. Observa-se que a temperatura máxima média e seu comparativo com a média do período de 1950-2019 manteve-se abaixo da média em todos os meses. No período de 2010-2020 a temperatura máxima anual foi de $28,9^{\circ} \mathrm{C}$ e suas oscilações mensais fluíram entre $25,3^{\circ} \mathrm{C}$ no mês de julho a $31,4^{\circ} \mathrm{C}$ em dezembro. A média climática do referido período em estudo oscilou com temperatura máxima anual de $29^{\circ} \mathrm{C}$ e suas oscilações mensais registrou-se com $25,4^{\circ} \mathrm{C}$ em julho a $31,5^{\circ} \mathrm{C}$ em dezembro. Na temperatura média das médias destacam-se suas flutuações mensais oscilando entre $19,4^{\circ} \mathrm{C}$ em julho a $23,4^{\circ} \mathrm{C}$ em dezembro e janeiro com 
temperatura média anual de $21,9^{\circ} \mathrm{C}$. Na variabilidade da temperatura média (1950-2020) a temperatura média foi de $22^{\circ} \mathrm{C}$ e as flutuações mensais registraram-se nos meses de julho com $19,5^{\circ} \mathrm{C}$ a $23,5^{\circ} \mathrm{C}$ nos meses de dezembro e janeiro.

Os meses mais frios foram maio a agosto e os quentes outubro a janeiro, este estudo vem a corroborar com os resultados apresentados por Buriol et al. (1987) onde mencionam os meses maio, junho, julho e agosto como os meses mais frios para Santa Maria.

Figura 8 - Temperatura decadal (2010-2020) e seu comparativo com a da série 1950-2020 em São Bento do Una - PE.

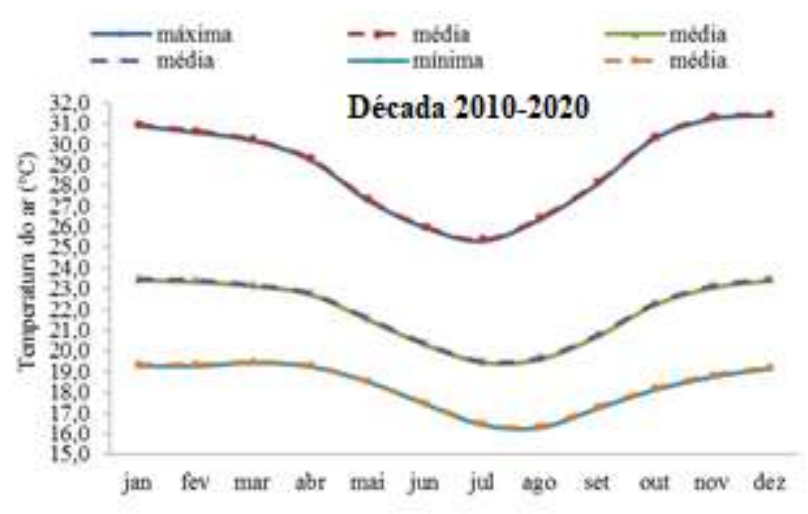

Fonte: Medeiros, (2021).

Como o Nordeste do Brasil (NEB), apresenta problemas com a falta e má distribuição dos postos meteorológicos, Medeiros et al. (2005), apud Bardin, Junior \& Morais (2010) estimaram e especializaram as temperaturas do ar (máxima, mínima e média) dessa região. Os coeficientes de determinação dos valores mensais e anuais de temperatura máxima, os coeficientes de determinação oscilaram de 0,85 (julho) a 0,52 (fevereiro) e, para os valores de temperatura mínima, de 0,85 (abril) a 0,64 (novembro) (Bardin et al. 2010). Para as temperaturas máximas médias mensais, a componente altitude teve significância de 5\% de probabilidade, exceto nos meses maio, junho e julho. Porém, a latitude e a longitude não foram significativas em todos os meses, de acordo com estudos de Pezzopane et al. (2004), apud , Junior \& Morais. (2010) que encontraram resultados aproximadamente semelhantes, e observaram que a variável longitude não foi significativa para o Estado do Espírito Santo.

\section{Conclusão}

Registram-se nas décadas de 1950, 1970, 1980, 1990 e 2000 aumentos nas três temperaturas estudadas. Na década de 1960 ocorreram reduções nos três elementos. 2010 as temperaturas se equipararam com as temperaturas climáticas.

As variabilidades observadas nas temperaturas decadais não estão interligadas as flutuações dos sistemas de meso e grande escala atuantes no período estudado.

Os dados e as variabilidades de suas oscilações na temperatura do ar auxiliam as tomadas de decisões no meio agropecuário, urbano e rural em múltiplas áreas do conhecimento.

A temperatura média é a representação da variabilidade e suas oscilações das temperaturas extremas, qualquer variabilidade nestes elementos as temperaturas médias se adequam as suas oscilações e decorrem dos sistemas sinóticos atuantes na época do período chuvoso e seco tal como dos impactos no meio ambiente e a sua elevação em relação ao nível do mar. 
Research, Society and Development, v. 10, n. 4, e40110413726, 2021

(CC BY 4.0) | ISSN 2525-3409 | DOI: http://dx.doi.org/10.33448/rsd-v10i4.13726

Variabilidades climáticas registradas em escala global, regional e local foram constatadas na área estudada, no período de sessenta e seis anos (1950-2020).

As variabilidades de aumento na temperatura média do ar são devido à forte tendência de elevação da temperatura mínima do ar e das estabilidades nas suas oscilações mensais, anuais e decadais ora fluindo com reduções e aumentos podendo caracterizam tendência a possíveis mudanças climáticas.

A elevação e a latitude são as variáveis fisiográficas que explicam melhor a variação da temperatura do ar anual e que as variabilidades da temperatura média.

A distribuição espacial da temperatura máxima, média e mínima apresentou variabilidade para ambos os meses e décadas estudadas, com variação de $0,1^{\circ} \mathrm{C}$ a $0,2^{\circ} \mathrm{C}$ nas suas décadas.

\section{Referências}

Alvares, C. A., Stape, J. L., Sentelhas, P. C., Gonçalves, J. L. M., \& Sparovek, G. (2014). Köppen's climate classification map for Brazil. MeteorologischeZeitschrift 22, 711-728.

Araújo, G. P., \& Gonçalves, F. L. T. (1998). Análise climatológica preliminar de eventos extremos de frio no Parque Estadual das Fontes do Ipiranga IAG/USP. In: Congresso.

Barbarisi, B. F., Pilau, F. G., Marin, F. R., Assad, E. D., \& Pinto, H. S. (2006). Estimativa da temperatura do ar para os Estados de Mato Grosso do Sul, Mato Grosso, Goiás e Tocantins a partir do uso de imagens de radar. In: Congresso Brasileiro de Meteorologia, 14, 2006, Florianópolis,

Bardin, L., Júnior, M. J. P., \& Moraes, J. F. L. (2010). Estimativa das temperaturas máximas e mínimas do ar para a região do Circuito das Frutas, SP. Revista Brasileira de Engenharia Agrícola e Ambiental, 14(6), 618-624, 2010.

Bettolli, P. O. (2018). Statistical downscaling of daily precipitation and temperatures in southern La Plata Basin. International Journal of Climatology, 38, $3705-3722$.

Buriol, G. A. et al. (1987). Análise das temperaturas mínimas diárias no município de Santa Maria-RS. I - Cálculo dos valores médios e da variabilidade, registro de valores extremos e comparação de periodicidade dessas temperaturas. In Congresso Brasileiro de Agrometeorologia, 5, 1987, Belém. Anais... O clima e o desenvolvimento rural brasileiro, Belém, p. 240-242.

Climate Research Unit. Global temperature record. http://www.cru.uea.ac.uk/cru/info/warming.

Campos, C. G. C. (2010). Padrões climáticos atuais e futuros de temperatura do ar na região sul do Brasil e seus impactos nos cultivos de pêssego e nectarina. 2010. Tese (Doutorado em Meteorologia) - Instituto Nacional de Pesquisas Espaciais, São José dos Campos, 2010.

Cavalcanti, E. P., Silva, V. P. R., \& Sousa, F. A. S. (2006). Programa computacional para a estimativa da temperatura do ar para a região Nordeste do Brasil. Revista Brasileira de Engenharia Agrícola e Ambiental, 10 (1), 140-147.

Cavalcanti, E. P., \& Silva, E. D. V. (1994). Estimativa da temperatura do ar em função das coordenadas locais. In: Congresso Brasileiro de Meteorologia, 8, 1994. Belo Horizonte, Anais... SBMET, 1, 154-157.

Coelho, C., Oliveira, C., Ambrizzi, T., Reboita, M., Carpenedo, C., Campos, J., Tomaziello, A., Pampuch, L., Custódio, M., Du tra, L., Rocha, R., \& Rehbein, A. (2016). The 2014 southeast Brazil austral summer drought: regional scale mechanisms and teleconnections. Climate Dynamics 46, $3737-3752$.

Collins, J., Chaves, R., \& Marques, V. (2009). Temperature Variability over South America. Journal of Climate 22, 5854-5869.

Cordeiro, A., Berlato, M., Fontana, D., \& Alves, R. (2016). Tendências climáticas das temperaturas do ar no Rio Grande do Sul, Sul do Brasil. Revista Brasileira de Geografia Física 9, 868-880.

, J., Loikith, P., Pampuch, L., Mechoso, C., Barkhordarian, A., \& Lee, H, (2019). Characterizing monthly temperature variability states and associated meteorology across southern South America. International Journal of Climatology 40, 492-508, https://doi.org/10.1002/joc.6224.

Feitoza, L., Scárdua, J., Sediyama, G., \& Valle, S. (1980). Estimativas das temperaturas médias das máximas mensais e anual do Estado do Espírito Santo. Revista do Centro Ciências Rurais, Santa Maria, 10, 25-32.

IDMC. Centro de Monitoramento de Deslocamento Interno 2017.

Intergovernamental Panel on Climate Change - IPCC: Climate Change 1996: Impacts, Adaptations and Mitigation of Climate Change. Cambridge Univ. Press.

Intergovernamental Panel on Climate Change - IPCC: Climate Change 2001: The Scientific basis IPCC WG. Cambridge Univ. Press.

Intergovernmental Panel on Climate ChangE - IPCC. Climate Change 2007: The Physical Science Basis. Cambridge: University Press. 
Research, Society and Development, v. 10, n. 4, e40110413726, 2021

(CC BY 4.0) | ISSN 2525-3409 | DOI: http://dx.doi.org/10.33448/rsd-v10i4.13726

Intergovernmental Panel on Climate Change. IPCC. 2014: Impacts, Adaptation, and Vulnerability. Part B: Regional Aspects. Working Group II Contribution to the Fifth. Assessment Report of the Intergovernmental Panel on Climate Change.

Kayano, M., Andreoli, R., Souza, R., \& Garcia, S. (2017). Spatiotemporal variability modes of surface air temperature in South America during the 19512010

Köppen, W., \& Geiger, R. Klimate Der Erde. Gotha: Verlag Justus Perthes. (1928). Wall-map 150cmx200cm.

Lopart, M., Reboita, M., Coppola, E., Giorgi, F., Rocha, R., \& Souza, D. (2018). Land Use Change over the Amazon Forest and Its Impact on the Local Climate. Water 10. 10.3390/w10020149.

Marengo, J., \& Silva, D. P. (2006). Mudanças climáticas globais e seus impactos nos recursos hídricos. Capitulo 3 em Águas Doces do Brasil: Capital Ecológico, Uso e Conservação, p.63-109, Eds. A. Rebouças, B., Braga e J. Tundisi. Editoras Escrituras, SP.

Matos, R. M., Medeiros, R. M., Silva, P. F., \& Saboya, L. M. F. (2015). Flutuabilidade da temperatura média do ar anual, máximos e mínimos absolutos no município de Barbalha - CE, Brasil In: Congresso Técnico Científico de Engenharia e da Agronomia, 2015, Fortaleza - Ceará. Anais Congresso Técnico Científico de Engenharia e da Agronomia. v.1.

Medeiros, R. M., Kozmhinsky, M., Holanda, R. M., \& Silva, V. P. (2018). Temperatura média do ar e suas flutuações no Estado de Pernambuco, Brasil. Revista Brasileira de Meio Ambiente, 2(1). 081-093.

Medeiros, R. M., \& Cavalcanti, E. P. (2020). Tendência climática das temperaturas do ar no município de Bom Jesus do Piauí, Brasil. Research, Society and Development, 9(7):1-23, e315973882.

Medeiros, R. M., Holanda, R. M., Viana, M. A., \& Silva, V. P. (2018). Climate classification in Köppen model for the state of Pernambuco - Brazil. Revista de Geografia (Recife). 35, 219 - 234.

Medeiros, R. M., (2018). Bom Jesus do Piauí - Brasil e suas flutuações nas amplitudes térmicas mensal, anual e dos trimestres quentes e frios. Revista Científica Rural, Bagé-RS, volume1, ${ }^{\circ} 1$.

Medeiros, R. M. (2016). Estudo agroclimático do Estado do Pernambuco. P.136.

Medeiros, R. M., Borges, C. K., \& Gomes Filho, M. F. (2014). Variabilidade e Mudanças Climáticas em Séries de Temperatura Máxima do Ar, Umidade Relativa do Ar e Precipitação em Brasília-DF. Revista Brasileira de Geografia Física. 7(2), 211-222.

Medeiros, R. M., Tavares, A. L., Kassar, C. B., Silva, J. A. S., \& Silva, V. P. R. (2012). Metodologias de cálculo da temperatura média diária do ar: aplicação para os municípios de parnaíba, picos e gilbués, pi. Revista Brasileira de Agricultura Irrigada. 6, 283 - 295.

Medeiros, S. S., Cecílio, R. A., Melo Júnior, J. C. F., \& Silva Júnior, J. L. C. (2005). Estimativa e espacialização das temperaturas do ar mínimas, médias e máximas na região Nordeste do Brasil. Revista Brasileira de Engenharia Agrícola e Ambiental, 9(2), 247-255.

Melo, V. S., Medeiros, R. M., \& Sousa, F. A. S. (2015). Flutuabilidade da temperatura máxima e mínima do ar mensal e anual de 10 municípios do estado de Pernambuco In: Congresso Técnico Científico de Engenharia e da Agronomia, 2015, Fortaleza - Ceará. Anais Congresso Técnico Científico de Engenharia e da Agronomia. 2015. v.1.

Montroull, N., Saurral, R., \& Camilloni, I. (2018). Hydrological impacts in La Plata basin under 1.5, 2 and $3{ }^{\circ} \mathrm{C}$ global warming above the preindustrial level. International Journal of Climatology 38, 3355-3368.

Oliveira, P., Santos, S. M., \& Lima, K. (2017). Climatology and trend analysis of extreme precipitation in subregions of Northeast Brazil. Theoretical and Applied Climatology 130, 77-90.

Oliveira Neto, S. N., Reis, R. G., Reis, M. G. F., \& Leite, H. G. (2000). Estimativa de temperaturas para o território brasileiro localizado entre 16 e $24^{\circ}$ latitude Sul e $48^{\circ}$ e $60^{\circ}$ longitude Oeste. In: Congresso Brasileiro de Meteorologia, 926-931.

Pezzopane, J. R. M., Santos, E. A., Eleutério, M. M., Reis, E. F., \& Santos, A. R. (2004). Espacialização da temperatura do ar no Estado do Espírito Santo. Revista Brasileira de Agrometeorologia, 12(1), 151-158.

Reguero, B., Losada, I., \& Méndez, F. (2019). A recent increase in global wave power as a consequence of oceanic warming. Nature Communications 10: https://doi.org/10.1038/s41467-018-08066-0.

Rossato, M. S. (2011). Os climas do Rio Grande do Sul: variabilidade, tendências e tipologia. 240 f. Tese (Doutorado) - Universidade Federal do Rio Grande do Sul. Instituto de Geociências. Programa de Pós-Graduação em Geografia.

Silva, R. G. (2000). Introdução á bioclimatologia animal. Nobel. 2000.

Vincent, L. A. et al. (2005). Observed trends in indices of daily temperature extremes in South America 1960-2000. Journal of Climate, Boston, 18, 50115023 . 